\title{
Classification of non stationary signals using multiscale decomposition
}

\author{
Marwa Chendeb $^{1,2}$, Mohamad Khalil ${ }^{2 *}$, David Hewson ${ }^{1}$, Jacques Duchêne ${ }^{1}$
}

${ }^{1}$ ICD, FRE CNRS 2848, University of Technology of Troyes, Troyes, France;

${ }^{2}$ LaSTRe Laboratory, Engineering Section 1, Lebanese University, Tripoli, Lebanon.

Email: ${ }^{*}$ mkhalil@ieee.org

Received 15 July 2008; revised 5 December 2009; accepted 11 December 2009.

\begin{abstract}
The aim of this article is to develop an automatic algorithm for the classification of non stationary signals. The application context is to classify uterine electromyogram (EMG) events to prevent the onset of preterm birth. The idea is to discriminate between the events by allocating them to the physiological classes: contractions, foetus motions, Alvarez or Long Duration Low Frequency waves. Our method is based on the Wavelet Packet (WP) decomposition and the choice of a best basis for classification purpose. Before classification, there is a need to detect events in the recorded signals. The discrimination criterion is based on the calculation of the ratio between intra-class variance and total variance (sum of the intra-class and inter-class variances), calculated directly from the coefficients of the selected WP. We evaluated the performance of the algorithm on real signals by using the classification methods Neural Networks (NN) and Support Vector Machines (SVM). Subband energies of the best selected WP are used as effective features. The determined best basis is applicable to a wide range of uterine EMG signals from large range of patients. In most cases, more than $85 \%$ of events are well classified whatever the term of gestation.
\end{abstract}

Keywords:Uterine EMG; Preterm Birth; Wavelet Packet; Best Basis; Event Classification

\section{INTRODUCTION}

The automatic classification of non stationary signals is an important studied problem especially as the nonstationarity precludes classification in the time or frequency domain [1]. The aim of this paper is to use the nonparametric representation wavelet packet transform (WPT) which is suitable for nonstationary signals and choose among the wavelet packets (WPs) the best basis for classification. The application context is the classification of uterine electromyographic (EMG) events used for the prevention of preterm birth. The progress of labour can be assessed non-invasively using EMG signals from the uterus (the driving force for contractility) recorded from the abdominal surface $[2,3]$.

Preterm labour and resultant preterm birth are the most important problems in perinatology $[2,4,5]$. Knowledge of labor commencement, as well as the possible prediction of its starting time, would be of great interest in terms of limiting unnecessary stays in hospitals and adapting treatment to the actual state of the pregnancy. The principal events extracted from the relevant activities of uterine EMG are the contractions (CT). Other events can be of value for pre-term birth diagnosis: Alvarez (Alv) waves, foetus motions (MAF) and long-duration low-frequency (LDBF) waves [2] (Figure 1). Several works have been carried out on mammals, with electrodes placed on the uterine surface $[2,5]$. They demonstrated a modification in electrical activity during both preterm and term labor. In [6] the uterine EMG signals are classified using artificial neural networks method to distinguish the normal term labour from abnormal preterm labour signals. [7] applied the wavelet transform on the uterine signals recorded using abdominal surface electrodes. In literature, the best basis algorithm is used to find the best-adapted WP for a lot of goals such the detection [8,9], denoising [10], feature extraction and classification [11,12], etc. Saito and Coifman introduced the Local Discriminant Bases (LDB) to search a best basis for classification [12]. Wavelet packet analysis is used to extract the features of the sample DNA sequences in [13]. An index of discrimination based on Kullback-Leibler distance is proposed as a way to select most discriminant wavelet packets for texture classification in an image [14].

In this work, classification of uterine EMG events by allocating them to the physiological classes: CT, MAF, Alv, or LDBF waves.is based on their energy distribution throughout the wavelet packet transform (WPT) which is used because it is characterized by the frequency 

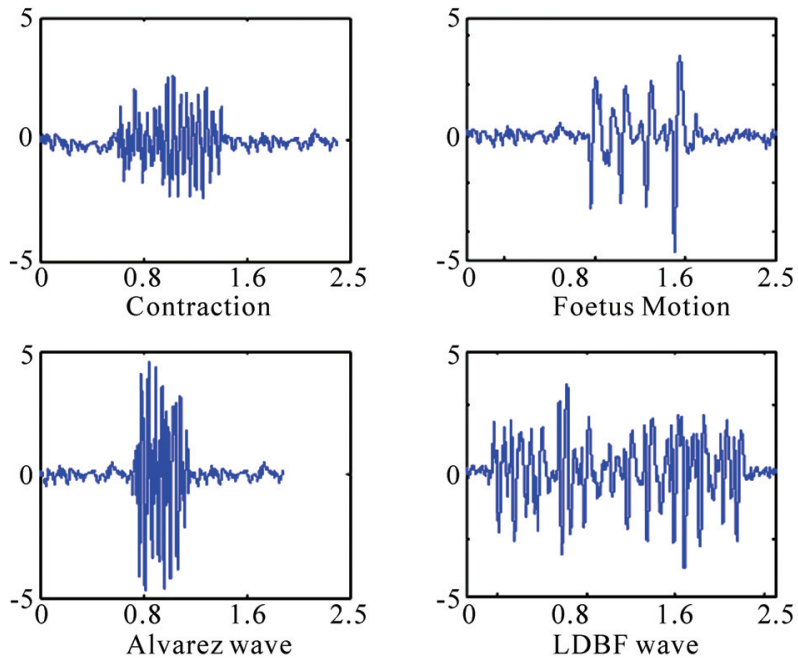

Figure 1. Samples of various events appearing in the uterine EMG recordings. $\mathrm{X}$ axis: minutes; $\mathrm{Y}$ axis: Amplitude scale in arbitrary units.

content of the packets $[2,3]$. Only a few WP of the redundant tree is relevant for classification purpose to define the features of events. The idea behind WP selection (best basis for classification) is to define an index for discrimination purpose. The ratio (for each WP) between intra-class and total variances (intra-class and inter-class variances), calculated directly from the wavelet packet coefficients (WPC) is the proposed discrimination criterion. An additional characteristic, the duration of the events, is also taken into consideration, as previous studies have shown its importance in terms of discrimination [3].

This paper is organized as follows. In Section 2, the signal composition and the used data are presented. In Section 3, WP decomposition is briefly described. The detection step is mentioned in Section 4. The index discrimination, the best basis for classification and the classification methods are displayed in Section 5. The performance of the method using real datasets is shown in Section 6. Discussion and conclusion are in Sections 7 and 8 respectively .

\section{SIGNAL DESCRIPTION}

\subsection{Signal Composition}

Uterine EMG signals are the electromyographic (EMG) activity of the uterus during labor recorded using abdominal electrodes placed across the maternal abdomen. Recordings were carried out in Amiens hospital setting under the supervision of the research group of Pr. Catherine Marque at the University of Technology of Compiègne, France $[2,10]$. They can be described by a random process that is composed of the EMG signal, the superimposed events and the noise due to the environment, especially electrode and instrumentation noise.
When the uterus contracts, an electrical activity is generated and the contractions can characterize the uterus states. The superimposed signals correspond to short potentials or artifacts which appear randomly throughout the signal, such as foetus motions, Alvarez waves and other superimposed events $[2,3,15]$. Alvarez waves appear during the first 30 weeks of the human pregnancy. Other waves have been recently discovered, such as LDBF waves, whose impact on obstetrical diagnosis has not yet been clearly identified. The evoked events present different time and frequency features deduced from spectral analyses. At mi term, the frequency of contractions is less than $0.2 \mathrm{~Hz}$ and at late term, it is greater than $0.5 \mathrm{~Hz}$. Alvarez frequency band is $0.2 \mathrm{~Hz}$ to $1 \mathrm{~Hz}$. MAF frequency is less than $0.5 \mathrm{~Hz}$ and LDBF waves have a very low frequency [2]. The contractions and the Alvarez events have the same frequency contents but the length of a contraction is greater than that of an Alvarez wave. The same interpretation can be used for the LDBF events and the foetus motion related events (very short) (Figure 1). The normalized amplitude is used throughout the paper.

Classical signal pre-processing includes the necessary step of signal to noise ratio (SNR) improvement. The denoising techniques based on the wavelet packet transform are widely used $[8,16]$. In the present work, the selection of a specific WP subset (best basis) automatically improves the SNR by keeping only the WPs containing the useful uterine information.

\subsection{Data Description}

The group named (CLASS) was defined in order to test classification efficiency. It contains 100 real events of each class (CT, Alv, MAF, LDBF, and noise) identified by an expert. Half of them are belonging to the training set (train_CLASS), the others are belonging to the test set (test_CLASS).

The acquired initial real uterine EMG signals were amplified and filtered between 0.2 and $6 \mathrm{~Hz}$ to eliminate the continuous component and the artefacts due to powerline interference. The sampling frequency was set to $\mathrm{Fe}=16 \mathrm{~Hz}$.

\section{WAVELET PACKET TRANSFORM}

WPT is an extension of Discrete Wavelet Transform (DWT) and can be obtained by a generalization of the fast pyramidal algorithm [17]. It enables non-stationary signals with different frequency features to be distinguished. Each detail and approximation coefficient's vectors are filtered and down-sampled using $l o(n)$ and $h i(n)$, the two impulse responses of low-pass and high-pass analyzing filters. Each node is associated with a subspace $\Omega_{j, n}$ generated by an orthonormal basis $\left\{\psi_{\mathrm{j}, \mathrm{n}}\right\}_{n \in \mathrm{Z}}$, with $\mathrm{j}$ being interpreted as a scale parameter, 
and $n$ as a sequence parameter. The wavelet packet coefficients (WPC) at each node $(j, n)$ are computed as [17]:

$$
\left(C_{j, n}(k)\right)=\left\langle f(t), \psi_{j, n}\left(t-2^{j} k\right)\right\rangle
$$

where $\mathrm{f}(\mathrm{t})$ is the initial signal and $\mathrm{k}$ is the time-localization index. In the following, each WP is characterized by the sequential index $\mathrm{v}$ obtained by according sequential numbers to the WP (see Figure 2). In fact, WPC carry the same information as the reconstructed signals. As WPT is a linear transformation, WPC exhibit the same statistical properties as the initial signal [8]. Consequently, WPC of the real noise follow the normal distribution [15].

\section{DETECTION}

To detect events, several methods can be used. In our last work [15] an algorithm of best basis for detection is proposed. It is based on the Kullback Leibler distance as a criterion to select the best WPs which show clearly the events. After choosing the best basis, the detection algorithm DCS (Dynamic Cumulative Sum) is applied on every selected wavelet packet coefficients. After the delay correction and the change time fusion [15], the uterine events of the real signals are obtained.

\section{CLASSIFICATION}

After change detection, the problem consists in identifying the detected events by allocating them to physiological classes: contractions, foetus motions, Alvarez waves, LDBF waves, or noise. In this section, the classification criterion is described. The approach of best basis is proposed for the classification task. The principal features for the classification methods are the variances of the selected packets. The duration of events was introduced as an additional feature to improve the correct classification rate (CCR).

\subsection{Classification Criterion}

An important criterion to discriminate between classes is to minimise the intra class variance and to maximise inter-class variance. In [18] an index which maximises the Euclidian distance between the classes was used. The most discriminant coefficients of all packets were selected as a criterion for classification [19]. In our work, the ratio between intra-class variance and total variance (sum of inter-class and intra-class variances) calculated for each WP seems to be an efficient index for discrimination. As the uterine EMG events are characterised by their frequency content, the variances of selected WPs produce useful information to identify events.

For the level of decomposition $\mathrm{J}$, the number $\mathrm{N}$ of packets is:

$$
N=2^{J+1}-1
$$

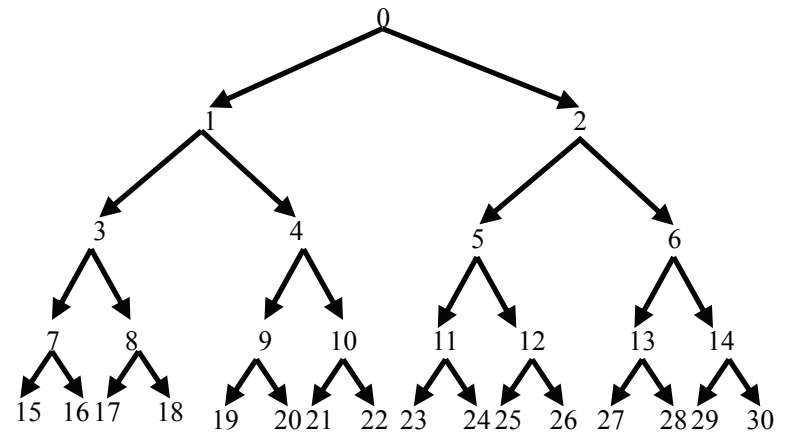

Figure 2. Wavelet packet decomposition tree.

Suppose that the ith class is composed of mi elements, so the gravity center of this class is:

$$
\hat{g}_{i}^{v}=\frac{1}{m_{i}} \sum_{q=1}^{n_{i}} x_{i q}^{v}
$$

where $x_{i q}^{v}$ is the qth element of class $\mathrm{i}(\mathrm{i}=1.5)$ and WP number $\mathrm{v}$.

The gravity center global of WP $\mathrm{v}$ is:

$$
\hat{g}=\frac{1}{m} \sum_{q=1}^{M} m_{q} \hat{g}_{q}^{v}
$$

where $\mathrm{M}$ is the number of classes (in our case $\mathrm{M}=5$ ) and $\mathrm{m}$ is the total number of samples.

The intra-class variance $\hat{\Sigma}_{w}^{v}$ of a WP number $\mathrm{v}$ is defined as:

$$
\hat{\Sigma}_{w}^{v}=\frac{1}{m} \sum_{i=1}^{M} \sum_{q=1}^{m_{i}}\left(x_{i q}^{v}-\hat{g_{i}^{v}}\right)^{2}
$$

where $g_{i}^{v}$ is the center of gravity of the packet $v$ and class $i$, mi the number of elements of class $i, M$ the total number of classes and $x_{i q}^{v}$ is the qth element of the packet $\mathrm{v}$ and class $i$.

The inter-class variance $\hat{\Sigma}_{B}^{v}$ of WP $\mathrm{v}$ is written as:

$$
\hat{\Sigma}_{B}^{v}=\frac{1}{m} \sum_{i=1}^{M} m_{i}\left(\hat{g_{i}}-\hat{g}^{v}\right)^{2}
$$

where $g^{v}$ is the centre of gravity for all classes.

The total variance $\hat{\Sigma}$ is equal to the sum of the inter-class and intra-class variances [20]:

$$
\hat{\Sigma}^{v}=\hat{\Sigma}_{w}^{v}+\hat{\Sigma}_{B}^{v}
$$

For each packet $v$, define the discrimination criterion as follows:

$$
R^{v}=\frac{\hat{\Sigma_{w}}}{\hat{\Sigma}}
$$




\subsection{Best Basis Selection for Classification}

The goal of this part is to retain only the wavelet packets that are able to discriminate between events in a specific class of signals (uterine EMG in our application). If the distances between classes are important and each class is strongly concentrated, the ratio $\mathrm{R}^{\mathrm{v}}$ of packet $\mathrm{v}$ is small. In this case, the classes are well separated and the packet is one of the best WPs for classififcation.

The values of criterion $\mathrm{R}^{\mathrm{v}}$ are calculated for all WP and sorted in ascending order. There is a clear gap between the values of $\mathrm{R}^{\mathrm{v}}$ corresponding to the packets that are relevant for classification and the others (Figure 3), a threshold can be indicated. As the tree is highly redundant, there is a need for further step to reduce the number of selected packets based on the frequency contents of events. In our case, the packets which contain the bandwidths of the events are retained as best basis for classification. The variances of the retained WPs (best basis) are used as features for the classification methods.

\subsection{Classification Methods}

Classification has been achieved using the two methods: neural networks (NN) [21] and Support Vector Machines (SVM) $[22,23]$.

\subsubsection{Neural Networks}

The Multi Layer Perceptron (MLP) is widely used to solve classification problems using supervised training for instance, the feed forward technique, which is used to minimize error. A multilayer perceptron is a feedforward artificial neural network model that maps sets of input data onto a set of appropriate output [24].

Such a network is based on the calculation of the output (direct calculation, weights are fixed) and adjustment of the weight by minimizing an error function. The process continues until the outputs of the network

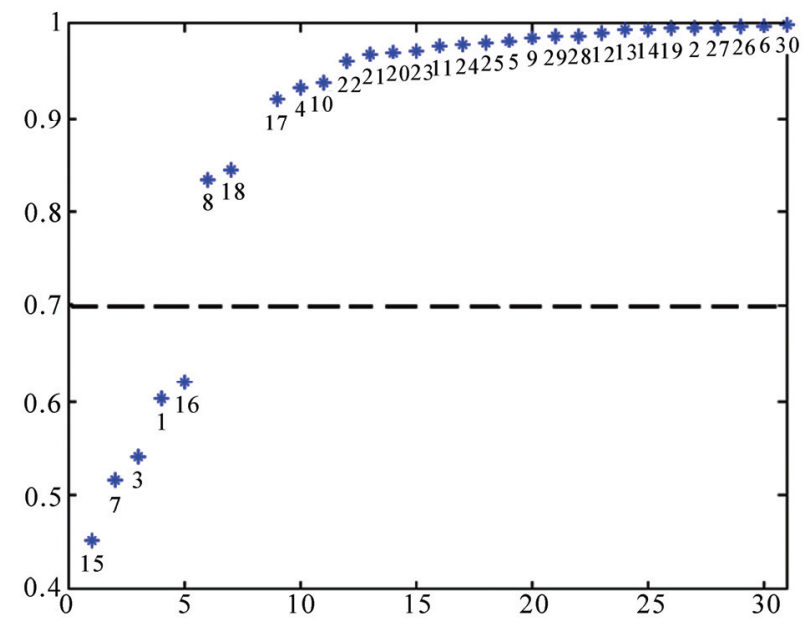

Figure 3. $\mathrm{R}^{\mathrm{v}}$ values of each WP plotted in ascending order. $\mathrm{X}$ axis: arbitrary units. $\mathrm{Y}$ axis: $\mathrm{R}^{\mathrm{v}}$ values. be come close to those desired. The network is defined by the transfer function of the neuron, the number of layers and the number of neurons in each layer. The number of inputs is equal to the dimension of the input vectors (in this case the variances of the selected packets). The number of outputs depends on the number of classes. Various transfer functions (sigmoid, hyperbolic tangent, linear, etc) can be used as neural activation functions [24].

\subsubsection{Support Vector Machines}

The SVM method is a new discriminator based on the construction of an optimal hyperplane, which is constructed in such a way that it maximizes the minimal distance between itself and the learning set.

Support vector machine is a learning technique which is well-founded in modern statistical learning theory [22]. It uses the training data to create the optimal separating hyperplane between two classes. The optimal hyperplane maximizes the margin of the closest data points. In this way the SVM minimizes the misclassification probability of new cases. The optimal separating hyperplane is computed as a decision surface of the form:

$$
g(\mathrm{y})=\operatorname{sgn}\left(\sum_{i=1}^{l_{s}} d_{i} \alpha_{i} K\left(\mathrm{y}_{i}, \mathrm{y}\right)+b\right)
$$

where $\mathrm{x}_{\mathrm{i}}$ are support vectors which are determined from the training data, $K\left(\mathrm{y}_{i}, \mathrm{y}\right)$ is the inner product kernel which must satisfy Mercer's theorem [22], and is used to map the data from its original dimension to higher dimension so that the data is linearly separable in the mapped dimension, $1_{\mathrm{s}}$ is the number of support vectors, $\mathrm{d}_{\mathrm{i}}$ is the class indicator $\left(d_{i} \in\{-1,+1\}\right)$ of $\mathrm{x}_{\mathrm{i}}$, and $\mathrm{b}$ is bias. The coefficients $\alpha_{i}$ are calculated by solving the quadratic programming problem:

Maximize $\sum_{i=1}^{l} \alpha_{i}-\frac{1}{2} \sum_{i, j=1}^{l} d_{i} d_{j} \alpha_{i} \alpha_{j} K\left(y_{i}, y_{j}\right)$

Subject to $\sum_{i=1}^{l} \alpha_{i} d_{i}=0, \quad 0 \leq \alpha_{i} \leq C \quad$ for $\quad i=1, \ldots, l$

where $\mathrm{C}$ is a user specified positive regularization parameter used to control he amount of allowed overlap between classes. Given the expression of $\mathrm{g}(\mathrm{y})$, the decision is based on the sign of $\mathrm{g}(\mathrm{y})$. In this work a radial basis function (RBF) is chosen as inner product kernel, which is defined as:

$$
K(u, v)=\exp \left(-\frac{\|u-v\|^{2}}{2 \sigma^{2}}\right)
$$

where $\sigma \succ 0$ is a user specified constant which defines the kernel width. In RBF kernel support vector machine, number and value properties of support vectors deter- 
mine the number of kernels and their centers [8]. Using $\mathrm{RBF}$ as an inner product kernel provides classification of a non-linear set of data, which means perfect discrimination of the prostate texture features.

To discriminate between the various uterine events, the SVM multiclass method [23] was used. It is based on building a model SVM for each group of events, enabling discrimination in comparison to the other groups.

\section{RESULTS ON CLASSIFICATION}

As the main discriminant feature of the events contained in uterine EMG recordings is the frequency content, the decomposition was performed up to level 4 . The used wavelet is symlet 5 [15]. For this, every event was decomposed onto $30 \mathrm{WP}$ (Figure 2). This choice is justified by the fact that the level 4 is the limit where WP still contain relevant information related to all the uterine events. For example, the WPs 15 and 16 correspond to the frequency bands which belong MAF and LDBF waves.

In detection case, the decomposition level is limited to 3 because the goal was to choose the best packets for detection whatever the events [15]. To decide which packets were able to classify the events, the values Rv of the discrimination criterion were computed on each packet using equation 8 (there are 30 packets). The events of train_CLASS were used (see Subsection 2.2).

Figure 3 shows the criterion values in ascending order. The selection of the discriminant WP was made by applying a threshold and selecting those WP which have the smallest criterion values.

By examining Figure 3 there is a clear threshold for Rv. In first step, the packets $1,3,7,15$ and 16 were selected. These packets correspond to the bandwidths $[0$, $4],[0,2],[0,1],[0,0.05]$ and $[0.05,1] \mathrm{Hz}$, respectively. The second step consists in eliminating the redundant packets by keeping only the packets which correspond to the frequency contents of events. As the contractions and Alvarez waves have a frequency band less than $1 \mathrm{~Hz}$, MAF frequency is less than $0.5 \mathrm{~Hz}$ and LDBF waves have a very low frequency (see Subsection 2.1), only the packets 7,15 and 16 are retained as best basis for classification.

In order to demonstrate that $\mathrm{Rv}$ is a good criterion to discriminate between uterine classes, the validation step is carried out. The classification methods MLP and SVM were applied on the test_CLASS to evaluate the Correct Classification Rate (CCR).

In the current application, a two layer feed forward network is created. The first layer has five tansig $\left(f(n)=\frac{2}{1+e^{-2 n}}-1\right)$ neurons, the second layer has ten pure$\operatorname{lin}(f(n)=n)$ neurons.

For the SVM method, the CCR were calculated for some kernels (linear, polynomial, sigmoid and Gaussian
Radial Basis Function (GRBF)) [22]. The kernel GRBF gave the best CCR, it is defined as follows [22]:

$$
K(u, v)=\exp \left(-\frac{\|u-v\|^{2}}{2 \sigma^{2}}\right)
$$

To provide the reader with the performance improvement of the best basis with respect to the DWT, we also present the CCR produced by using the packets $2,4,8$ and 16 corresponding to the DWT. The variances of packets $2,4,8$ and 16 were used as features for the classification methods. The best basis method was evaluated by comparing the results with those obtained using the DWT. Results are summarized in Tables 1,2, showing the performance of the use of the variances of the selected WP as features for the classification methods. Table 1 shows the CCR of MLP classifier for the best basis selection and DWT. The CCR were calculated for large values of the regularization parameter $\mathrm{C}$ and $\sigma$ for the SVM method $[22,23](\mathrm{C}=[0.0001,0.001,0.01,0.1$, $1,10,100,1000,10000$, Infinity $]$ and $\sigma=[0.1,0.2,0.4$, $0.6,1,5,10])$. C controls the tradeoff between the complexity of the machine and the number of non- separable points. $\mathrm{C}$ and $\sigma$ are chosen for each class in such a way that the best correct classification rates are obtained. The optimal values of $\mathrm{C}$ and $\sigma$ are presented in Table 2 for the best basis selection and DWT.

Event duration is used as an additional feature to improve the correct classification rate. The correct classification and false alarm rates are presented in Table 3 after including the event durations in the case of best basis. The results are well improved specifically for Alvarez and MAF waves.

\section{DISCUSSION}

This paper developed a best basis selected from the set of WP tree for classification. The uterine EMG events are characterized by their frequency content justify the use of WPT. WP tree reduction is generally guided by a certain criterion (depending on the WPT objectives) based on the knowledge of a data (often statistical) model or the availability of training data. The mother wavelet choice in the current work was based on the minimum delay induced by applying detection algorithms directly on the wavelet packet coefficients. The choice was made using all available EMG events, leading to the selection of the Symlet 5 wavelet [15]. This choice is probably due to the symmetrical shape of the associated filters. It is better to study the influence of

Table 1. Correct classification rates in the cases of best basis (WP: 7, 15 and 16) and DWT (WP: 2, 4, 8 and 16) for MLP method.

\begin{tabular}{cccccc}
\hline SVM & CT & ALV & MAF & LDBF & Noise \\
\hline best basis & 0.80 & 0.80 & 0.86 & 0.88 & 0.94 \\
DWT & 0.52 & 0.62 & 0.62 & 0.66 & 0.94 \\
\hline
\end{tabular}


Table 2. Correct classification rates in the cases of best basis (WP: 7, 15 and 16) and DWT (WP: 2, 4, 8 and 16) for SVM method.

\begin{tabular}{cccccc}
\hline SVM & CT & ALV & MAF & LDBF & Noise \\
\hline \multirow{2}{*}{ best basis } & 0.82 & 0.64 & 0.8 & 0.88 & 0.82 \\
& $\underline{\sigma}=5, \mathrm{C}=\mathrm{Inf}$ & $\underline{\sigma}=10, \mathrm{C}=\mathrm{Inf}$ & $\underline{\sigma}=5, \mathrm{C}=\mathrm{Inf}$ & $\underline{\sigma}=1, \mathrm{C}=\mathrm{Inf}$ & $\underline{\sigma}=5, \mathrm{C}=100$ \\
DWT & 0.54 & 0.50 & 0.60 & 0.50 & 0.90 \\
& $\underline{\sigma}=1, \mathrm{C}=10$ & $\underline{\sigma}=0.2, \mathrm{C}=\mathrm{Inf}$ & $\underline{\sigma}=0.2, \mathrm{C}=\operatorname{Inf}$ & $\underline{\sigma}=0.4, \mathrm{C}=10000$ & $\underline{\sigma}=0.6, \mathrm{C}=1000$ \\
\hline
\end{tabular}

Table 3. Correct classification probabilities (a) and false alarm rates (b) for the two methods after including event duration (best basis case).

\begin{tabular}{ccccccccccc}
\hline \multirow{2}{*}{ Method } & \multicolumn{2}{c}{ CT } & \multicolumn{2}{c}{ ALV } & \multicolumn{2}{c}{ MAF } & \multicolumn{2}{c}{ LDBF } & \multicolumn{2}{c}{ Noise } \\
\cline { 2 - 12 } & (a) & (b) & (a) & (b) & (a) & (b) & (a) & (b) & (a) & (b) \\
\hline MLP & 0.86 & 0.04 & 0.96 & 0.11 & 0.98 & 0 & 1.00 & 0.12 & 0.86 & 0.04 \\
\multirow{2}{*}{ SVM } & 0.88 & 0.04 & 0.86 & 0.12 & 0.96 & 0 & 0.98 & 0.03 & 0.90 & 0.18 \\
& $\underline{\sigma}=5, \mathrm{C}=$ Inf & \multicolumn{2}{c}{$\sigma=5, \mathrm{C}=1$} & \multicolumn{2}{c}{$\sigma=5, \mathrm{C}=1$} & $\sigma=10, \mathrm{C}=1$ & $\sigma=0.6, \mathrm{C}=1$ \\
\hline
\end{tabular}

other wavelets to choose the best wavelet for classification.

The best basis was searched for classification. Four levels were needed as they corresponded to the relevant bandwidths for event discrimination such as foetus motions and LDBF waves.

The ratio between intra-class and inter-class variances appeared as a good discrimination criterion for the choice of the best basis for the classification of uterine EMG events. The best basis for classification was selected by choosing all packets that scored a ratio lower than a defined threshold as explained in Section 4. A second step based on the frequency contents of events is introduced to eliminate the redundant packets. In order to ensure the performance of the selection of best basis, we asked an expert in uterine EMG events to indicate, from an arbitrary data set, which WP could discriminate between the uterine events at best. She selected the same WP obtained by our algorithm.

This result illustrates the coincidence between the automatic unsupervised learning and direct supervised selection. Two classification methods (Neural Networks (MLP) and Support Vector Machines) were applied on validation and test data. The main issues related to the use of MLP were the choice of the activation functions of the hidden and output layers and the definition of the number of hidden neurons. Results were satisfactory with or without the use of the event duration as a complementary feature.

For SVM method, the parameter $(\mathrm{C}$ and $\sigma)$ values that produced the best CCR were selected. These values were different according to whether the duration of the events was used or not. The CCR were greatly improved by the introduction of the event duration, whatever the classification method.

\section{CONCLUSIONS}

The method proposed for event classification in uterine recordings based on a WPT, and best basis selection in order to reduce the WP tree produced very satisfactory results. The ratio between intra-class and total variance was found to be a good criterion well adapted for the choice of the best discriminant packets. Two proposed classifiers (Neural Networks and SVM) for the identification of the detected events by allocating them to physiological classes (CT, MAF, Alv or LDBF waves) were used. On average more than $85 \%$ of the events were correctly classified, regardless of the pregnancy term. The training data permits to choose the best basis relevant to the uterine EMG events but the algorithm can be used for other similar situations. As perspectives the study must continue in order to show the performance of the algorithm when applying it to other non stationary data.

Uterine electrical activity is increasingly used as a relevant index for the characterization of the uterine contraction within the scope of pregnancy and parturition monitoring. A further step would be the production of a sufficiently large database to improve the current knowledge on the actual recording contents and their correlation to a diagnosis of possible premature birth.

\section{REFERENCES}

[1] Gretton, A. et al. (2001) Nonstationary signal classification using support vector machines. 11th IEEE Workshop on Statistical Signal Processing Proceedings, pp. 305-308.

[2] Devedeux, D., Marque, C., Mansour, S., Germain, G., Duchene, J. (1993) Uterine electromyography: a critical review. Amer. J. Obstet. Gynecol. 169(6), 1636-1653.

[3] Khalil, M., Duchene, J. (2000) Uterine EMG analysis: a dynamic approach for change detection and classification. IEEE Trans. Biomed. Eng, 47(6), 748-756.

[4] Garfield, R.E., Saade, G., Buhimschi, C., Buhimschi, I. et al. (1998) Control and assessment of the uterus and cervix during pregnancy and labour. European Society of 
Human and Embryology, 4(5), 673-695.

[5] Doret, M., Bukowski, R., Longo, M., Maul, H., Maner, W.L., Garfield, R.E. and Saade, G.R. (2005) Uterine electromyography characteristics for early diagnosis of mifepristone-induced preterm labor. Obstetrics and $G y$ necology, 15(4), 822-830.

[6] Lu, N., Wang, J., McDermott, I., Thornton, S. et al. (2009) Uterine electromyography signal feature extraction and classification. International Journal of Modelling, Identification and Control, 6(2), 136-146.

[7] Eswaran, H., Wilson, J.D., Murphy, P., Preissl, H., Lowery C.L. (2002) The Journal of Maternal-Fetal \& Neonatal Medicine, 11(3), 158-166.

[8] Ravier, P. and Amblard, P.O. (2001) Wavelet packets and de- noising based on higher-order-statistics for transient detection. Signal Processing, 81(9), 1909-1926.

[9] Hitti, E. and Lucas, M.F. (1998) Wavelet-packet basis selection for abrupt changes detection in multicomponent signals. Proc. EUSIPCO, Island of Rhodes, Greece, 1841-1844.

[10] Leman, H. and Marque, C. (2000) Rejection of the maternal electrocardiogram in the electrohysterogram signal, IEEE Trans Biomed Eng. 47(8), 1010-1017.

[11] Yang, B., Yan, G., Yan, R., Wu, T. (2007) Adaptive subject-based feature extraction in brain-computer interfaces using wavelet packet best basis decomposition. Medical Engineering \& Physics, 29(1), 48-53.

[12] Saito, N. and Coifman, R.R. (1994) Local discriminant bases. A. F. Laine and M. A. Unser Editors, Wavelet Applications in Signal and Image Processing \{II\}, Proc. SPIE 2303, 2-14.

[13] Zhao, J., Yang, X.W., Li, J.P. and Tang, Y.Y. (2001) DNA sequences classification based on wavelet packet analysis from "wavelet analysis and its applications book".
Springer Berlin / Heidelberg editor.

[14] Rajpoot, N.M. (2002) Texture classification using discriminant wavelet packet subbands. 45th Midwest Symposium on Circuits and Systems, 3, 300-303.

[15] Chendeb, M., Khalil, M. and Duchêne J. (2006) Methodology of wavelet packet selection for event detection. Signal Processing, 86, 3826-3841.

[16] Nadernejad, E. (2008) $\mu$ PET data de-noising using wavelet packets. Contemporary Engineering Sciences, 1(2), 91-104.

[17] Mallat, S. (1999) a wavelet tour of signal processing, Academic Press, San Diego, CA.

[18] Sheng, S., Yanda, L. (1997) "Best Bases for Classification". Technical Report of pattern Recognition and Signal Processing Lab, department of Automation, Tsinghua University.

[19] Fukunaga, K. (1990) "Introduction to statistical pattern recognition". Academic Press, Inc.

[20] Dubuisson, B. (1990) "Diagnostic et reconnaissance des formes". Editions Hermes.

[21] Turkoglu, I., Arslan, A. and Ilkay, E. (2003) An intelligent system for diagnosis of the heart valve diseases with wavelet packet neural networks. Computers in Biology and Medicine, 33(4), 319-331.

[22] Gunn, S.R. (1998) Support vector machines for classification and regression. Technical Report, School of Electronics and Computer Science, University of Southampton.

[23] Mayoraz, E. and Alpaydin, E. (1998) Support vector machines for multi-class classification. Technical Report IDIAP.

[24] Haykin, S.S., Gwynn, R. (2008) Neural networks and learning machines. Prentice Hall, 3rd edition. 